\title{
Ultra-fast, high density 3D mapping (Rhythmia Mapping System) and catheter radiofrequency ablation for electrical storm - early single centre experience
}

Radosław Lenarczyk ${ }^{1, \mathrm{~A}-\mathrm{F}}$, Patrycja Pruszkowska, ${ }^{1, \mathrm{~B}-\mathrm{C}, \mathrm{E}-\mathrm{F}}$, Stanisław Morawski ${ }^{1, \mathrm{~A}-\mathrm{C}, \mathrm{E}-\mathrm{F}}$, Michał Mazurek ${ }^{1, \mathrm{D}-\mathrm{F}}$, Beata Średniawa ${ }^{2, A, E-F}$, Adam Sokal $^{1, C, E-F}$, Ewa Jędrzejczyk-Patej ${ }^{1, A-F}$, Monika Koziel $^{1, C, E-F}, Z^{1}$ bigniew Kalarus ${ }^{2, A, C, E-F}$, Oskar Kowalski ${ }^{1, \mathrm{~A}, \mathrm{C}, \mathrm{E}-\mathrm{F}}$

A - Research concept and design, B - Collection and/or assembly of data, C - Data analysis and interpretation,

D - Writing the article, E - Critical revision of the article, F - Final approval of article

1 Department of Cardiology, Congenital Heart Disease and Electrotherapy, Silesian Center for Heart Disease

2 Department of Cardiology, Congenital Heart Disease and Electrotherapy, Silesian Medical University, Silesian Center for Heart Disease

Address for correspondence:

Radosław Lenarczyk, Department of Cardiology, Congenital Heart Disease and Electrotherapy, Silesian Center for Heart Disease email: radle@poczta.onet.pl

Patrycja Pruszkowska, Department of Cardiology, Congenital Heart Disease and Electrotherapy, Silesian Center for Heart Disease email: patrycja.pruszkowska@interia.pl

Stanisław Morawski, Department of Cardiology, Congenital Heart Disease and Electrotherapy, Silesian Center for Heart Disease email: morawski.stan@gmail.com

Michał Mazurek, Department of Cardiology, Congenital Heart Disease and Electrotherapy, Silesian Center for Heart Disease email: m.i.c.h.a.l@wp.pl

Beata Średniawa, Department of Cardiology, Congenital Heart Disease and Electrotherapy, Silesian Medical University, Silesian Center for Heart Disease email: bms@pro.onet.pl

Adam Sokal, Department of Cardiology, Congenital Heart Disease and Electrotherapy, Silesian Center for Heart Disease email: asokal20@gmail.com

Ewa Jędrzejczyk-Patej, Department of Cardiology, Congenital Heart Disease and Electrotherapy, Silesian Center for Heart Disease email: ewajczyk@op.pl

Monika Koziel, Department of Cardiology, Congenital Heart Disease and Electrotherapy, Silesian Center for Heart Disease email: kozielmonika@poczta.fm

Zbigniew Kalarus, Department of Cardiology, Congenital Heart Disease and Electrotherapy, Silesian Medical University, Silesian Center for Heart Disease email: zbigniewkalarus@kalmet.com.pl

Oskar Kowalski, Department of Cardiology, Congenital Heart Disease and Electrotherapy, Silesian Center for Heart Disease email: oskowa@vp.pl

Received: 23.12 .2016

Revised: 28.12 .2016

Accepted: 28.12.2016 


\section{Abstract}

Background. Electrical storm (ES) is a life-threatening condition, and catheter ablation can be curative in this syndrome. The Rhythmia Mapping System (RMS, Boston Scientific) is a novel system that allows for ultra-fast, high-density 3D mapping. The aim of this study was to assess the feasibility, safety and short-term effectiveness of catheter ablations performed with the RMS for ES.

Materials and methods: Ablations for ES in five patients who underwent the first mapping using the RMS system at our centre (2016) were compared with the procedures in a cohort of 56 consecutive patients mapped for ES with the Carto system (Biosense Webster) between 2010 and 2015.

Results: Time to construct RMS maps was $41 \mathrm{~min}$ and the average map had 8776 points (214 points per minute). Procedure duration (160 $\mathrm{min}$ ), fluoroscopy duration (23 $\mathrm{min}$ ) and radiologic exposure (333 mGy) were significantly (all $\mathrm{p}<0.05)$ greater in RMS procedures. They were however similar (all $\mathrm{p}=\mathrm{NS}$ ) to procedural parameters during the first 5 Carto procedures in 2010. Arrhythmia mapping was possible in $80 \%$ of RMS patients and in $27 \%$ of Carto procedures ( $p=0.01$ ). Acute success ( $100 \mathrm{vs} 91 \%$ ) and complication rates (0 vs 7\%) and short-term recurrence rates within a 2-month follow-up period ( $20 \mathrm{vs} .32 \%$, all $\mathrm{p}=\mathrm{NS}$ ) were similar in RMS and Carto-mapped subjects.

Conclusions: Mapping and ablation of ES with the RMS are comparably safe, feasible and effective as Carto procedures. Longer procedure duration and higher fluoroscopy burden associated with the RMS are typical for early stages of the learning curve. The RMS may be more effective in mapping during arrhythmia.

\section{Key words}

catheter ablation, basket mapping catheter, electrical storm, $3 \mathrm{~d}$ mapping systems, ventricular arrhythmia

\section{Background}

Electrical storm (ES) - an electric instability manifesting as multiple malignant ventricular arrhythmias clustered within a short period of time (usually defined as $\geq 3$ episodes $/ 24 \mathrm{~h}$ ) - is a life-threatening condition. Nowadays, the perception of this syndrome is usually synonymous with multiple discharges of an implantable cardioverter-defibrillator (ICD). ${ }^{(1)}$ Catheter ablation can be curative in ES, but non- inducibility of clinical arrhythmia or haemodynamic instability due to ongoing arrhythmia makes the procedure challenging. Additionally, relatively poor resolution of maps created with standard 4-mm tipped ablation catheters may lead to insufficiently detailed identification of surviving muscular "islets", surrounded by areas of scar tissue. Importantly, some of these regions are electrical isthmuses and may play a role in arrhythmia origin. These drawbacks of existing mapping systems may be responsible for the limited efficacy and relatively high recurrence rate after ES ablation. The Rhythmia Mapping System (RMS, Boston Scientific, Cambridge, MA, USA) is a novel mapping system that allows for ultra-fast, high-density 3D mapping.(2) Utility of this system has been demonstrated in animal models and subsequently verified in a wide variety of human arrhythmias. Nonetheless, evidence on applicability of Rhythmia in ES is limited. The aim of this study was to assess the feasibility, safety and short-term efficacy of catheter ablations performed using the Rhythmia Mapping System for ES.

\section{Methods \\ Patients}

This analysis comprises five patients who underwent the first catheter ablation procedures using the Rhythmia Mapping
System for ES. All procedures were performed in September and October 2016. These patients were compared with a cohort of 56 consecutive patients who underwent radiofrequency current (RF) ablation with the Carto system (Biosense Webster, Diamont Bar, CA, USA) for ES between May 2010 and December 2015. All the procedures were performed at the same tertiary cardiology centre. One patient was lost to follow-up and consequently excluded from further analysis. This patient was transferred to our centre from a distant region of the country in 2015 for RF Carto ablation.

Baseline demographics and clinical characteristics of the study population are summarised in Table 1 . No differences were observed amongst the studied groups, except that valvular disease requiring surgical repair was more common in the Rhythmia cohort, and diabetes was less prevalent in the RF group. In all patients reversible causes of electrical storm such as ischaemia, hyperthyroidism or others were excluded prior to ablation.

\section{Ablation procedure}

All procedures were performed in a fasting, conscious state, under superficial sedation and with local anaesthesia. The only exceptions to this rule were patients $(n=2,3.6 \%$ of the Carto treated group) who required intubation and mechanical ventilation due to haemodynamic instability associated with ongoing ventricular arrhythmia, in which cases general anaesthesia was used. Depending on clinical characteristics of patients (and likelihood of tachycardia origin) along with ECG morphology and sequence of intracardiac signals during arrhythmia, the mapping was confined to either the left or the right ventricle, or involved both chambers. Technical details 


\begin{tabular}{|c|c|c|c|}
\hline & $\begin{array}{c}\text { Rhythmia } \\
\text { treated } \\
\text { group } \\
\mathrm{N}=5\end{array}$ & $\begin{array}{l}\text { Carto } \\
\text { treated } \\
\text { group } \\
\mathrm{N}=56\end{array}$ & p \\
\hline Age, years & $69(54-84)$ & $65(39-80)$ & 0.69 \\
\hline Male & $5(100)$ & 52 (93) & 0.54 \\
\hline Ischaemic HF & $4(80)$ & 49 (87) & 0.64 \\
\hline NYHA class & $2(2-4)$ & $2(1-3)$ & 0.69 \\
\hline Arterial hypertension & $3(60)$ & $34(61)$ & 0.97 \\
\hline Diabetes & $4(80)$ & $20(36)$ & 0.05 \\
\hline Atrial fibrillation & $3(60)$ & $23(41)$ & 0.42 \\
\hline Chronic pulmonary disease & 0 & $5(9)$ & 0.49 \\
\hline Chronic kidney disease & $3(60)$ & $17(30)$ & 0.18 \\
\hline Prior CABG & 0 & $11(19)$ & 0.28 \\
\hline Prior valvular surgery & 1 (20) & $1(2)$ & 0.03 \\
\hline Haematocrit \% & $41(32-45)$ & $40.6(30-48)$ & 0.94 \\
\hline Serum creatinine $\mu \mathrm{mol} / \mathrm{L}$ & 146 (76-172) & 97 (57-183) & 0.13 \\
\hline QRS width ms & 127 (91-190) & 140 (78-262) & 0.56 \\
\hline LVEF \% & 30 (27-38) & $27(15-50)$ & 0.1 \\
\hline LVEDD mm & $65(53-79)$ & $68(51-82)$ & 0.77 \\
\hline Left atrium mm & $46(44-51)$ & $45(26-66)$ & 0.94 \\
\hline Severe mitral regurgitation & 0 & $1(2)$ & 0.76 \\
\hline ICD/CRT-D implanted & $60 / 40$ & $82 / 27$ & 0.24 \\
\hline \multicolumn{4}{|l|}{ Drugs at discharge } \\
\hline Beta-blocking agent & $5(100)$ & $56(100)$ & 0.99 \\
\hline ACE-I & $5(100)$ & $48(86)$ & 0.37 \\
\hline ARB & 0 & $5(9)$ & 0.49 \\
\hline MRA & $5(100)$ & $45(80)$ & 0.28 \\
\hline Digoxin & 0 & $6(10)$ & 0.44 \\
\hline Loop diuretic & $5(100)$ & $34(61)$ & 0.08 \\
\hline Amiodarone & $1(20)$ & $23(41)$ & 0.36 \\
\hline Other antiarrhythmic drugs & 0 & $6(10)$ & 0.44 \\
\hline \multicolumn{4}{|c|}{$\begin{array}{l}\text { Continuous variables were presented as median (range), dichotomic as numbers } \\
\text { (percent). Non-parametric tests were used for comparisons. Abbreviations: HF - } \\
\text { heart failure, NYHA - New York Heart Association, CABG - coronary artery bypass } \\
\text { grafting, LVEF - ejection fraction of the left ventricle, LVEDD - end-diastolic diameter } \\
\text { of the left ventricle, ICD - implantable cardioverter-defibrillator, CRT-D - cardiac } \\
\text { resynchronization pacemaker with cardioverter-defibrillator function, ACE-I - an- } \\
\text { giotensin-converting enzyme inhibitor, ARB - angiotensin receptor blocker, MRA } \\
\text { - mineralocorticoid receptor antagonist. }\end{array}$} \\
\hline
\end{tabular}

of the procedure were left to the discretion of the operator. Nonetheless, if the left ventricle was a target, the retrograde (transaortic) route/access was preferred, with the transseptal approach being usually reserved only for patients with aortic valve stenosis, artificial aortic valve or severe peripheral artery disease - Figure 1A. Tracings from a standard transthoracic

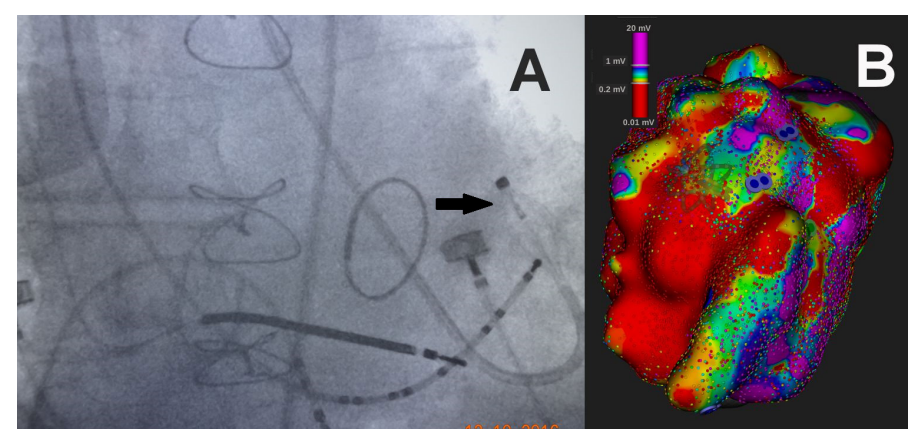

Figure 1. Chest X-ray and Rhythmia map in a patient undergoing ablation of electrical storm.

ECG lead, or a signal from the intracardiac electrode located in the right ventricle, served as an electric/time reference in patients in whom the Carto system was used; the intracardiac electrode provided an electrical and impedance reference in subjects mapped with the RMS. Type of ablation catheter used (standard 4-mm tip, cooled tip catheter, contact force measurement) was left to the discretion of the operator. A 64-electrode basket mapping electrode (Intelamap Orion, Boston Scientific) was used for mapping in the Rhythmia-mapped group, whereas the NaviStar, Biosense Webster ablation catheter was employed for the same purpose in the Carto group. A bolus of intravenous heparin was given to all patients immediately following sheath insertion, and subsequent doses of heparin were titrated to maintain ACT of $>250$ (with Carto) or $>300 \mathrm{~s}$ (with Rhythmia).

Irrespective of the system used, the initial step was to map the substrate. The principle was to create an anatomical shape of the chamber of interest, with the amplitude of intracardiac signals superimposed on the three-dimensional shell during sinus rhythm (or during pacing of the ventricle in pacemaker-dependent patients). The main goal of this part of the procedure was to identify any regions of scar tissue (amplitudes $\leq 0.2-0.5 \mathrm{mV}$; dense scar area was confirmed further by high-current pacing), as well as to delineate a normal, healthy myocardium (with signal amplitudes of $\geq 0.5-1.5 \mathrm{mV}$, depending on the mapping system) - Figure 1B. The remaining areas were marked as borderline and mapped further in a particularly detailed manner. Local signals indicating regions of slow conduction (local abnormal ventricular electrograms LAVA, or diastolic potentials) were marked on the map, as were signals suggesting lines of block (double potentials). ${ }^{(3)}$ For the RMS, a detailed inspection of signals from all surrounding electrodes was a key to clear and precise definition of the area of interest (i.e. assessment of the extent of diseased, slowly conducting myocardium). This allowed us to further narrow down the area of potentially critical channels. The relevance of these channels was subsequently confirmed by pacing. Stimulus-to-evoked potential delay and morphology match between paced ECG and clinical arrhythmia were considered strong indicators of pacing within the critical isthmus.

Having created an electro-anatomical map during sinus rhythm or stimulation, the second step involved arrhythmia provocation and its subsequent mapping. Such an approach was a standard procedure unless the patient became hemody- 
namically unstable or arrhythmia was non-inducible/non-sustained. The mapping during ongoing ventricular tachycardia (VT) allowed for identification of LAVAs/diastolic potentials and lines of block. Most importantly however, such a mapping technique makes it possible to build a precise activation map, thus allowing for identification of slow conduction zones and patterns of ventricular activation. Additionally, if possible, entrainment pacing was performed within slowly conducting areas, and concealed fusion and postpacing interval measurements facilitated further identification of critical isthmus. ${ }^{(4)}$

Eventually, RF applications were delivered in the regions of isthmus or, if critical areas could not be clearly delineated, within the areas of slow conduction, located in borderline zones. The end-point was non-inducibility of any arrhythmia (full success). Inducibility of non-clinical arrhythmia only was considered partial success of the procedure.

\section{Programming of the implantable devices and follow-up}

Between 2010 and 2012, ICDs in post-ablation patients were programmed using relatively low detection thresholds for VT $(>150 / \mathrm{min})$ and VF (>180/min). After the MADIT-RIT results were published, less aggressive settings were implemented for all devices (VT 170/min and VF 200-220/min), unless the patient presented with a slower $(<170 / \mathrm{min})$ but haemodynamically unstable VT, in which cases a second VT zone (with a longer detection cycle) was applied. ${ }^{(5)}$

After discharge, patients were followed up as outpatients after one month and subsequently every 6 (patients with resynchronisation devices) to 12 months (ICD patients). Apart from a routine clinical follow-up, arrhythmia status for all patients was continuously captured via device remote monitoring.(6) Adequate ICD intervention (high- or low-voltage) was considered arrhythmia recurrence. Criteria for arrhythmia recurrence were also met if sustained VT occurred, but resulted in no ICD response (e.g. due to the arrhythmia cycle length being too long for detection). To reduce the bias of between-group comparisons, a similar duration of follow-up period (of 2 months) was applied in both study groups (Rhythmia and Carto cohorts, respectively).

\section{Statistical analysis}

Continuous variables were presented as median (range), dichotomic ones as percent. The Mann-Whitney U-test was used for comparisons of continuous variables and Fisher's exact test for categorical variables. A $p$ value of $<0.05$ was considered significant.

\section{Results}

The median of 1 VT (range 1-2) with the cycle length of $330 \mathrm{~ms}$ (240-350) was targeted in the Rhythmia group. Time needed to construct Rhythmia maps was $41.02 \mathrm{~min}$ (30.01-66) and the average map consisted of 8776 points $(6205-18,354)$. Consequently, 213.9 points were collected per minute of mapping. For comparison, median procedure duration for 14 Carto procedures performed in 2015 was $132 \mathrm{~min}$ (85-235) and the average map consisted of 472 (142-4894) points (3.6 points collected per minute, $\mathrm{p}<0.001$ versus Rhythmia).

Procedural details and outcomes are shown in Table 2. The transseptal route to the left ventricle was more often used in

\begin{tabular}{|c|c|c|c|}
\hline & $\begin{array}{l}\text { Rhythmia } \\
\text { treated } \\
\text { group } \\
\mathrm{N}=5\end{array}$ & $\begin{array}{l}\text { Carto } \\
\text { treated } \\
\text { group } \\
\mathbf{N}=56\end{array}$ & p \\
\hline Procedure duration, min & $160(150-170)$ & $120(40-235)$ & 0.001 \\
\hline Fluoroscopy duration, min & $23(15-24)$ & $11.4(2.1-32)$ & 0.007 \\
\hline Fluoroscopy exposure, mGy & 333 (300-743) & 204 (18-1284) & 0.02 \\
\hline Transseptal approach & $3(60)$ & 3 (5) & $\begin{array}{c}\& l t ; 0.00 \\
1\end{array}$ \\
\hline Only left ventricle mapped & $5(100)$ & 50 (89) & 0.44 \\
\hline Both ventricles mapped & 0 & 4 (7) & 0.54 \\
\hline No. of VTs targeted & $1(1-2)$ & $1(1-4)$ & 0.87 \\
\hline Substrate mapping & $5(100)$ & 52 (93) & 0.54 \\
\hline Arrhythmia mapping & $4(80)$ & 15 (27) & 0.01 \\
\hline 4-mm tip ablation catheter & 0 & $17(31)$ & 0.15 \\
\hline Cool-tip catheter & $5(100)$ & 38 (69) & 0.15 \\
\hline $\begin{array}{l}\text { Ablation electrode with } \\
\text { contact force measurement }\end{array}$ & 0 & $8(15)$ & 0.37 \\
\hline \multicolumn{4}{|l|}{ Acute efficacy* } \\
\hline $\begin{array}{l}\text { Full success (no VT } \\
\text { induced) }\end{array}$ & $4(100)$ & $41(91)$ & 0.54 \\
\hline $\begin{array}{l}\text { Partial success } \\
\text { (no clinical VT) }\end{array}$ & 0 & $2(4.5)$ & 0.67 \\
\hline Failure (clinical VT induced) & 0 & $2(4.5)$ & 0.67 \\
\hline $\begin{array}{l}\text { No attempt to induce post } \\
\text { RFCA }\end{array}$ & $1(20)$ & 11 (19) & 0.98 \\
\hline $\begin{array}{l}\text { Procedure-related } \\
\text { complications }\end{array}$ & 0 & 4 (7) & 0.54 \\
\hline Vascular complication & 0 & 2 (3.5) & 0.67 \\
\hline $\begin{array}{l}\text { Hemodynamic } \\
\text { decompensation }\end{array}$ & 0 & $1(1.8)$ & 0.77 \\
\hline Other & 0 & $1(1.8)$ & 0.77 \\
\hline Arrhythmia recurrence & $1(20)$ & $18(32)$ & 0.58 \\
\hline \multicolumn{4}{|c|}{$\begin{array}{l}\text { Continuous variables are presented as median (range), dichotomic as numbers (per- } \\
\text { cent). Non-parametric tests were used for comparisons. *from among } 45 \text { patients in } \\
\text { whom attempts to induce arrhythmia were undertaken after ablation Abbreviations } \\
\text { VT - ventricular tachycardia, RFCA - radiofrequency catheter ablation. }\end{array}$} \\
\hline
\end{tabular}

the RMS group than in patients mapped with Carto (60 vs $5 \% \mathrm{p}<0.001)$. The remaining technical details and approaches were comparable in both groups. Procedure duration (160 vs $120 \mathrm{~min}$ ), fluoroscopy duration (23 vs $11 \mathrm{~min}$ ) and radiological exposure (333 vs $202 \mathrm{mGy}$ ) were significantly (all $p<0.05$ ) greater in patients in whom the Rhythmia system was used for mapping. However, when compared with the first 5 patients in whom the Carto procedure was performed (learning curve 
period - year 2010), procedure duration ( $\mathrm{p}=0.17)$, fluoroscopy duration $(\mathrm{p}=0.17)$ and exposure $(\mathrm{p}=0.6)$ are all similar to the RMS procedural data - Figure 2. While similar proportions

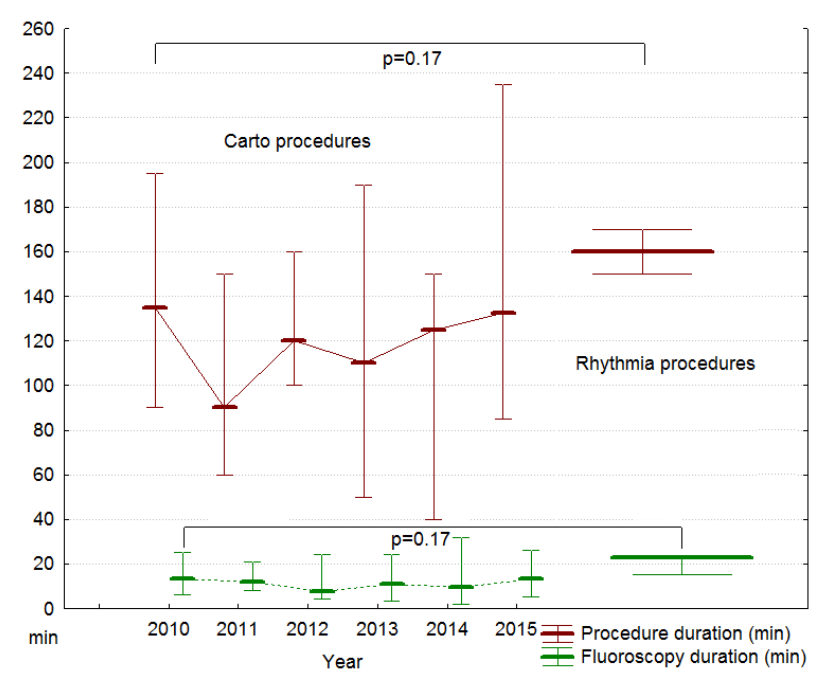

Figure 2. Procedure and fluoroscopy duration in patients undergoing ablation with Carto and Rhythmia systems

of patients from Rhythmia and Carto groups underwent successful substrate mapping $(100 \%$ and $93 \%$, respectively, $\mathrm{p}=0.54)$, detailed VT mapping $(>75 \%$ of arrhythmia cycle length) allowing a meaningful clinical interpretation was possible more often for Rhythmia than for Carto $(80 \%$ and $27 \%$, respectively, $\mathrm{p}=0.01$ ).

Procedure-related complication rates were $7 \%$ for Carto and $0 \%$ for Rhythmia $(\mathrm{p}=0.54)$. Vascular complications predominated: haematoma requiring antibiotic therapy and prolonged hospital stay in one patient, and arterio-venous fistula not requiring surgical intervention in another patient. In two other cases, haemodynamic instability necessitating inotropic support was noted, and transient bradycardia that was secured by temporary pacing.

Acute success rates were $100 \%$ and $91 \%$ for Rhythmia and Carto groups, respectively $(\mathrm{p}=0.54)$. The corresponding recurrence rates after a median follow-up of 2 months were $20 \%$ vs $32 \%$, respectively $(\mathrm{p}=0.58)$.

\section{Discussion}

Based on our preliminary data, the major findings of this study are as follows: 1) Safety and feasibility of procedures performed with the Rhythmia Mapping System for ES are similar to procedures performed with the Carto system; 2) early RMS-guided ablations are more patient- and operator-demanding, but procedure duration and fluoroscopic burden are similar to those observed for early procedures with the Carto system (which may be attributable to the learning curve); 3) the Rhythmia system may even be more effective than Carto, allowing for arrhythmia mapping during ongoing VT, possibly due to its high efficacy in map rendering; 4) direct post-procedural and short-term success rates of both mapping systems are similar.
The Rhythmia Mapping System has already been successfully used in various types of arrhythmias, including atrial tachycardia, atrial fibrillation, atrioventricular nodal re-entry tachycardia, ventricular tachycardia in structural heart disease or in the healthy heart ${ }^{(7-15)}$. The principal part of the mapping system is a $8.5 \mathrm{~F}$ basket mapping catheter (Intelamap Orion, Boston Scientific), introduced into the target chamber. The Orion mapping catheter is a deployable basket electrode (deployment range 3-22 $\mathrm{mm}$ ) equipped with 64 electrodes, thus capable of acquiring signals simultaneously from 64 different points of the cardiac cavity at one heartbeat. Each electrode has a size of $0.4 \mathrm{~mm} 2$ only, and with an inter-electrode distance of $2.5 \mathrm{~mm}$ this array creates a potential to generate very detailed (points are collected simultaneously by every electrode that is in contact with the myocardium), high-density maps (electrodes have small dimensions and are in close proximity to each other).

This feature can be particularly useful in severely compromised patients, such as subjects with heart failure, cardiac implantable devices and electric storm. In such patients, every possibility to speed up the process of creating a map can be crucial, as they may deteriorate rapidly unless the ongoing arrhythmia terminates. Viswanathan et al. showed that with the Rhythmia Mapping System the operator was able to create 25 VT maps in 19 patients, including 7 maps with $>90 \%$ activation captured. ${ }^{(16)}$ Similarly, we have shown that Rhythmia allows one to make a map during VT in a substantial proportion of ES patients, being significantly greater than with Carto. Although this feature did not translate into a higher success rate in our patients, one has to keep in mind that these are preliminary findings only. Also, the longer procedure duration and radiation burden have to be interpreted with caution. As we have shown, these figures are comparable with procedural parameters obtained in the early era of 3D ablation (namely, the Carto system in our series). However, the relatively high recurrence rates (20\% during 2 months) indicate that even with modern tools, such as the Rhythmia system, ablation in patients with electrical storm still remains a very challenging procedure.

\section{Conclusions}

Mapping and ablation of ES patients with the Rhythmia Mapping System is similarly safe, feasible and effective as procedures performed with the Carto system. Longer procedure duration and higher fluoroscopy burden associated with RMS use are typical for early stages of the learning curve. The Rhythmia system may be more effective than Carto in arrhythmia mapping during ongoing VT.

\section{Study limitations}

This is a non-randomised, observational study, with data coming from a single-centre registry. The Rhythmia-mapped group consisted of 5 patients only, and the learning curve has to be taken into consideration when analysing procedural data. The two groups differ with respect to some clinical characteristics (diabetes, prior valvular surgery), and procedural aspects 
(transseptal approach). The follow-up period is very short (2 months only). Therefore, this study has to be considered as hypothesis-generating only. Further research is required to evaluate the position of Rhythmia in daily clinical practice.

\section{References}

1. Kumar S, Fujii A, Kapur S, Romero J, Mehta NK, Tanigawa S, Epstein LM, Koplan BA, Michaud GF, John RM, Stevenson WG, Tedrow UB. Beyond the storm: comparison of clinical factors, arrhythmogenic substrate, and catheter ablation outcomes in structural heart disease patients with versus those without a history of ventricular tachycardia storm. J Cardiovasc Electrophysiol 2016, Published online ahead of print 2016 Oct 26. doi: 10.1111/jce.13117.

2. Thajudeen A, Jackman WM, Stewart B, Cokic I, Nakagawa H, Shehata M, Amorn AM, Kali A, Liu E, Harlev D, Bennett N, Dharmakumar R,Chugh SS, Wang X. Correlation of scar in cardiac MRI and high-resolution contact mapping of left ventricle in a chronic infarct model. Pacing Clin Electrophysiol 2015; 38: 663-74.

3. Jaïs P, Maury P, Khairy P, Sacher F, Nault I, Komatsu Y, Hocini M, Forclaz A, Jadidi AS, Weerasooryia R, Shah A, Derval N, Cochet H, Knecht S, Miyazaki S, Linton N, Rivard L, Wright M, Wilton SB, Scherr D, Pascale P, Roten L, Pederson M, Bordachar P, Laurent F, Kim SJ, Ritter P, Clementy J, Haïssaguerre M. Elimination of local abnormal ventricular activities: a new end point for substrate modification in patients with scar-related ventricular tachycardia. Circulation 2012; 125: 2184-96.

4. Stevenson WG, Friedman PL, Sager PT, Saxon LA, Kocovic D, Harada T, Wiener I, Khan H. Exploring postinfarction reentrant ventricular tachycardia with entrainment mapping. J Am Coll Cardiol 1997; 29: 1180-9.

5. Madit-RIT Moss A.J., Schuger C., Beck C.A. Reduction in inappropriate therapy and mortality through ICD programming. N Engl J Med. 2012;367:2275-2283.

6. Mazurek M, Jędrzejczyk-Patej E, Lenarczyk R, Liberska A, Przybylska-Siedlecka K, Kozieł M, Morawski S, Podolecki T, Kowalczyk J, Pruszkowska P, Pluta S, Sokal A, Kowalski O, Kalarus Z. Do we need to monitor the percentage of biventricular pacing day by day? Int J Cardiol. 2016 Oct 15;221:81-9.

7. Anter E, Tschabrunn CM, Contreras-Valdes FM, Li J, Josephson ME. Pulmonary vein isolation using the Rhythmia mapping system: Verification of intracardiac signals using the Orion mini-basket catheter. Heart Rhythm. 2015; 12: 1927-34.

8. Sohns C, Saguner AM, Lemes C, Santoro F, Mathew S, Heeger C, Reißmann B, Maurer T, Riedl J, Fink T, Hayashi K, Ouyang F, Kuck KH, Metzner A. First clinical experience using a novel high-resolution electroanatomical mappingsystem for left atrial ablation procedures. Clin Res Cardiol. 2016 Jun 11. [Epub ahead of print]
9. Tanaka Y, Genet M, Lee LC, Martin AJ, Sievers R, Gerstenfeld EP. Utility of high-resolution electroanatomic mapping of the left ventricle using a multispline basket catheter in a swine model of chronic myocardial infarction. Heart Rhythm 2015; 12: 144-154

10. Anter E, Tschabrunn CM, Buxton AE, Josephson ME. High-resolution mapping of postinfarction reentrant ventricular tachycardia: electrophysiological characterization of the circuit. Circulation 2016; 134: 314-27.

11. Latcu DG, Bun SS, Saoudi N. Combined remote magnetic navigation and ultra-high-density mapping (Rhythmia $^{\mathrm{TM}}$ ) in slow pathway ablation. Europace 2016; 18: 814. doi: 10.1093/europace/euv459.

12. Hooks DA, Yamashita S, Capellino S, Cochet H, Jais $\mathrm{P}$, Sacher F. Ultra-rapid epicardial activation mapping during ventricular tachycardia using continuous sampling from a high-density basket (Orion(TM)) catheter. J Cardiovasc Electrophysiol 2015; 26: 1153-4.

13. Anter E, McElderry TH, Contreras-Valdes FM, Li J, Tung P, Leshem E, Haffajee CI, Nakagawa H, Josephson ME. Evaluation of a novel high resolution mapping technology for ablation of recurrent scar-related atrial tachycardias. Heart Rhythm 2016; 13: 2048-55.

14. Steinberg BA,Piccini JP. High-density mapping of the tachycardia circuit in atrioventricular nodal reentrant tachycardia. Heart Rhythm Case Reports 2016; 2 :451453 ; DOI: http://dx.doi.org/10.1016/j.hrcr.2016.02.006

15. Lenarczyk R, Kowalski O, Mazurek M, Kalarus Z. Ultra-fast, high-density 3D mapping system for catheter ablation of infrequent, "unmappable" idiopathic ventricular arrhythmia - a case report. Heart Beat J 2016, Published online ahead of print 2016 December 21. doi: http://www.heartbeatjournal.com/Ultra-fast-high-density-3D-mapping-system-for-catheter-ablation-of-infrequent-unmappable-idiopathic-ventricular-arrhythmia-a-case-report-,67449,0,2.html

16. Viswanathan K, Mantziari L, Butcher C, Hodkinson E, Lim E, Khan H, Panikker S, Haldar S, Jarman JW, Jones DG, Hussain W, Foran JP, Markides V, Wong T. Evaluation of a novel high-resolution mapping system for catheter ablation of ventricular arrhythmias. Heart Rhythm. 2016: S1547-5271(16)31059-1. 